\title{
TRASPLANTE ALOGÉNICO EN LEUCEMIA MIELOIDE AGUDA Y LA IMPORTANCIA GENÉTICA EN DESARROLLO DE NUEVOS TRATAMIENTOS
}

\section{Allological transplant in acute myeloid leukemia, the genetic importance for the development of new treatments}

\author{
Manuela Arboleda-Rojas ${ }^{\mathrm{a}}$
}

Recibido: 12 marzo, 2021 • Aprobado: 6 junio, 2021

Cómo citar: Arboleda-Rojas M. Trasplante alogénico en leucemia mieloide aguda y la importancia genética en desarrollo de nuevos tratamientos. cysa [Internet]. [citado 22 de febrero de 2022];6(1):55-64. Disponible en: https://revistas.intec.edu.do/index.php/cisa/ article/view/2405

\section{Resumen}

La Leucemia Mieloide Aguda es una enfermedad caracterizada por la alteración en la producción de células madre hematopoyéticas y la proliferación celular. Es más común en adultos; a pesar de ello solo se presenta en el $1 \%$ en los Estados Unidos. Entre los 65-68 años se observa una mayor incidencia existiendo de 2-3 casos por cada año en 100.000 habitantes, siendo aproximadamente el $10 \%$ de los cánceres de este tipo. Los diagnósticos más recomendados para esta enfermedad son los de carácter sanguíneo, la realización de citometrías de flujo en muestra de médula ósea. Según estudios, los análisis citogenéticos en un gran número de pacientes han demostrado translocaciones e inversiones en los cromosomas somáticos, mientras que solo una minoría tiene una organización de cromosomas somáticos balanceada. La terapia de consolidación se acompaña del trasplante de células madre hematopoyéticas, conocido como el trasplante alogénico, que puede ser potencialmente curativo en algunos pacientes.

Palabras clave: leucemia mieloide aguda, células madres hematopoyéticas, intercambio genético, trasplante, injerto alogénico.

\begin{abstract}
The acute myeloid leukemia, is a disease which is a characterized by an irregular production of hematopoietic cells and cellular proliferation. It's most common in adults, however only $1 \%$ of American adults will be diagnosed throughout their lives. Between the ages of 65-68 there is a high incidence with only 2-3 cases per 100.000 patients; making up only $10 \%$ of this type of cancer. It's mainly diagnosed by using blood test, flow cytometry (on Bone Marrow samples). Some cytogenetic studies suggest that in a significant number of patients both somatic chromosomal inversion and translocation are present, while only a small percentage show no somatic chromosomal mutations. Consolidation therapy with a hematopoietic Stem Cells transplant, also known as a "allogenic transplant", can be potentially curative in some special cases.
\end{abstract}

Keywords: Acute myeloid leukemia, Hematopoietic Stem Cells, Crossing Over Genetic, transplant, Allografts.

\footnotetext{
a Grupo de investigación salud clínica y quirúrgica, Escuela de Ciencias de la Salud, Facultad de Medicina; Universidad Pontificia Bolivariana. Medellín. Antioquia. Colombia. ORCID: 0000-0002-4704-3855, Correo-e: manuela.arboledar@upb.edu.co
} 


\section{Introducción}

La producción de las células madre hematopoyéticas se da en la médula ósea; este proceso se caracteriza por ser complejo, debido a que las células germinales cumplen papeles importantes y muy específicos que dan lugar a la diferenciación celular a varias líneas celulares, como linfocitos, monocitos, células granulares (neutrófilos, eosinófilos, basófilos); es por esto que las células germinales hematopoyéticas son esenciales en el proceso de maduración de las células sanguíneas, estas se necesitan para los procesos de autorrenovación, correcto mantenimiento de la hematopoyesis y la pluripotencialidad, es decir, la capacidad que tiene una célula madre para dar origen a todas las células sanguíneas maduras ${ }^{1,2}$.

Cuando se presenta una anormalidad en este ciclo de producción se pueden dar diversas alteraciones como las leucemias, que existen de varios tipos; una de las más comunes son la (LMA), que es una enfermedad caracterizada por la alteración en la producción de células madre hematopoyéticas y variaciones en la regulación en los mecanismos de la proliferación celular ${ }^{3}$. La LMA, a pesar de ser considerada como una enfermedad infrecuente, es más común que se presente en adultos que en niños; según estudios realizados recientemente, se presenta en no más del $1 \%$ de adultos en los Estados Unidos. El diagnóstico de esta enfermedad es entre los 65-68 años, observándose una mayor incidencia después de la sexta década de vida ${ }^{4}$.

La supervivencia en los pacientes con leucemia mieloide aguda ha venido aumentando en las últimas décadas, gracias al perfeccionamiento en tratamientos, sin embargo, hay varios factores que influyen para que sea exitosa la recuperación de los pacientes, dentro de ellas se encuentran el estadio en el cual está la enfermedad, factores de riesgo relacionados con la mortalidad, poca adherencia al tratamiento y compli- caciones que se pueden ir presentando en el proceso de tratamiento de la LMA5.

Existe una incidencia de 2-3 casos por cada año en 100.000 habitantes, en el cual se observa una relación hombre:mujer de un $5: 3^{3}$, además, siendo aproximadamente el $10 \%$ de los casos de cánceres de este tipo ${ }^{6}$. En los Estados Unidos se observa una supervivencia a largo plazo baja; los datos de registro demuestran que solamente $27 \%$ de los pacientes con LMA están vivos a los cinco años ${ }^{7}$.

Según el Instituto Nacional de Cancerología de Colombia, en 2012, se presume que hubo, aproximadamente, 350.000 casos nuevos de leucemias en el mundo, lo que significa que aumentó en un $3 \%$ de los casos nuevos de cáncer. Según este reporte la incidencia más alta se ha observado en población blanca de los Estados Unidos y en Canadá y también se ha observado un mayor número de muertes cada ańo en unos 265471 casos reportados, representando cerca de $3 \%$ de las muertes por cáncer ${ }^{8}$. En la región de América Latina y El Caribe se presentan cerca de 29000 casos nuevos, los cuales indican el 2,7 \% del total de casos nuevos por LMA. En mujeres, las tasas de incidencia de leucemias más altas corresponden en Argentina, Brasil y Ecuador, mientras que en hombres están en Brasil, Colombia y Argentina?.

Desde el punto de vista colombiano, existen varios estudios que afirman una supervivencia libre de la enfermedad aproximadamente del $38 \%$, apoyados de otros estudios en los cuales la supervivencia global es de 13 meses promedio ${ }^{10}$, con una mortalidad después del trasplante para leucemia mieloide aguda del $26 \%$, obteniendo unos datos estadísticos de mejor sobrevida, pero a pesar de esto, se deben seguir investigando posibles tratamientos que aumenten la supervivencia en los pacientes de LMA. El pronóstico para esta alteración es bueno, siempre 
y cuando se diagnostique en fases iniciales y haya buena adherencia al tratamiento ${ }^{11}$.

\section{Materiales y métodos}

Este estudio es una revisión de tema, de la especialidad de medicina interna, hematología.

La investigación fue realizada entre los meses de noviembre de 2019 a marzo de 2020, en donde se consultó y revisaron artículos originales, artículos de revisión, revisiones de tema, reportes de casos en las bases de datos Science Direct, ClinicalKey, Scielo y PubMed. Los términos de búsqueda utilizados en español fueron leucemia mieloide aguda, tipos de leucemias con el booleano [AND] nuevos tratamientos, mientras que las palabras de búsqueda para el idioma inglés fueron acute myeloid leukemia. El número de artículos hallados en español fue de aproximadamente 200 artículos, y en inglés se encon- traron alrededor de 76.900 artículos, dentro de los cuales se seleccionaron 50. La estrategia mayormente utilizada por el autor fue leer detenidamente los títulos de los artículos, el año de la publicación y lo más importante, mirar el resumen para darse cuenta de si ese escrito servía como referente para dar respuesta al objetivo del trabajo, o eran pobres en información relevante como definición de la enfermedad, aspectos genéticos, nuevos tratamientos, criterios diagnósticos, entre otros. Se excluyeron todos aquellos artículos que tuvieran año de publicación antes del 1998, por contenido de poca validez, falta de acceso al texto completo y donde la información fuera casi la misma. Se incluyeron artículos con años de publicación entre 1998 y 2019; siendo la mayoría de las publicaciones en los últimos años (entre 5 a 7 años), artículos que tuvieran información clara y concisa que cumplieran con el objetivo del trabajo y aquellas que contaran, en especial, con el abordaje de nuevos tratamientos para la LMA.

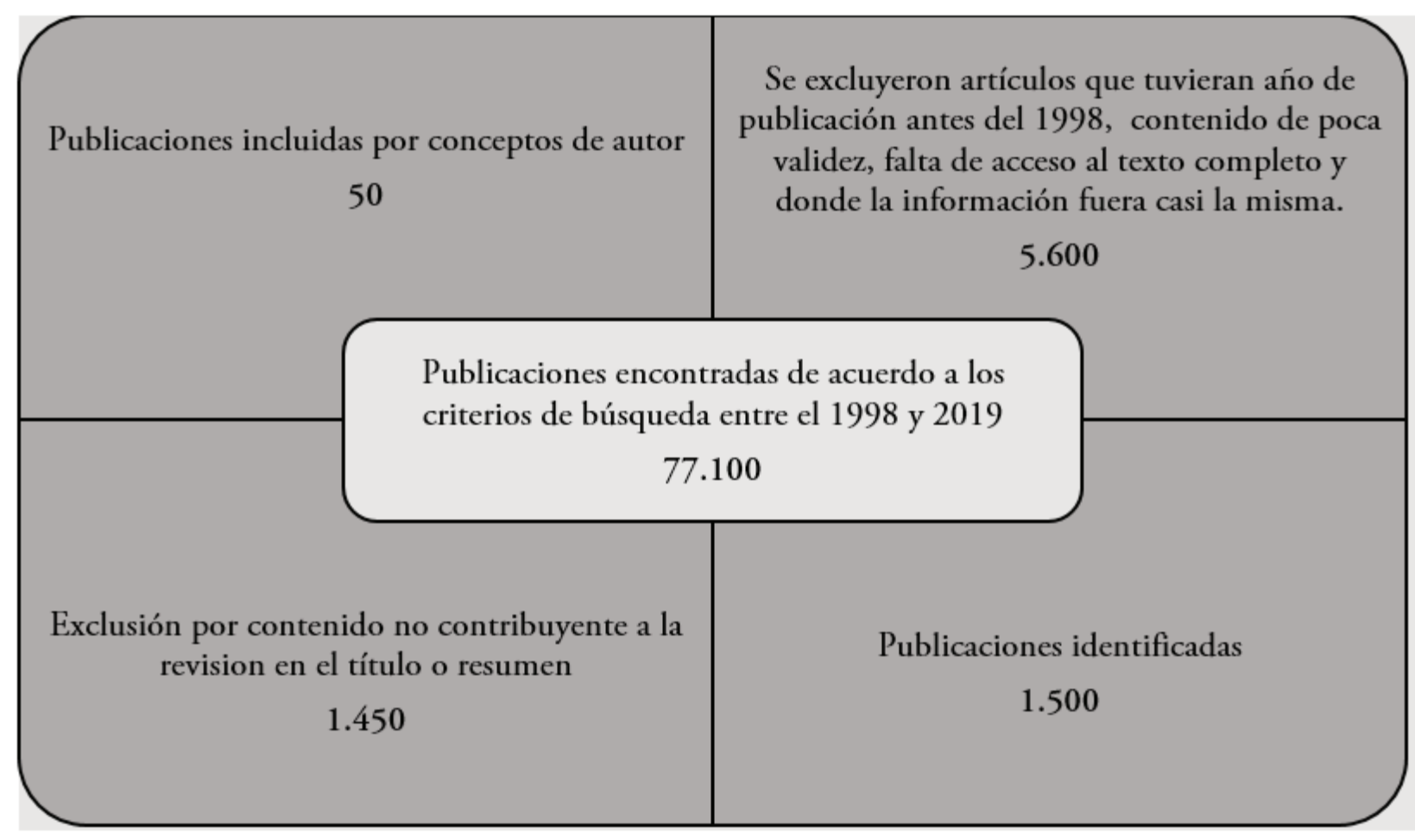

Figura 1. Criterios de inclusión y exclusión en metodología de búsqueda.

Fuente: elaboración propia. 


\section{Discusión}

\section{Factores de riesgo}

La LMA es una enfermedad idiopática, que no es precedida por alteraciones de la médula ósea, sin embargo, puede darse el caso de que la larga exposición a algunos agentes tóxicos y radiaciones de gran intensidad, influyan en el desarrollo de la enfermedad, además de que los tratamientos previos con quimioterapia y/o radioterapia podrían contribuir también al desarrollo de la enfermedad, pero no de manera significativa. Adicionalmente, se debe tener en cuenta que la LMA puede tener factores de riesgo importantes como: la edad, recuento de leucocitos, inmunofenotipo ${ }^{12}$, así como el componente genético observado gracias a diferentes anormalidades en los cromosómicas somáticos, como las translocaciones y la diferente relación con la presentación clínica de la enfermedad ${ }^{13,14}$.

\section{Presentación clínica}

Las manifestaciones clínicas son de fundamental importancia para el conocimiento de la LMA. Entre las manifestaciones más frecuentes, en primer lugar, se hallan en un $66,7 \%$ la palidez mucocu- tánea, seguido de la fiebre, astenia y adinamia en un $33,3 \%$, luego, con un $22,2 \%$ de incidencia, la pérdida de peso, adenopatías y, por último, con un valor del $11,1 \%$, se presentan las manifestaciones dérmicas, en las cuales se pueden presentar varios tipos de lesiones dermatológicas que pueden ayudar a orientar el diagnóstico de la hemopatía maligna; dentro de las más comunes están las lesiones cutáneas específicas, lesiones "satélites", infecciones cutáneas y lesiones secundarias a la quimioterapia ${ }^{15}$. También se observaron algunos casos de pacientes que no presentaron síntomas iniciales; todo lo anterior puede ser apoyado para identificar la enfermedad mediante los cambios en la morfología de las células hematopoyéticas ${ }^{16,17}$.

Las leucemias agudas son el producto final de una alteración en cromosomas somáticos, en donde una célula madre hematopoyética desencadena una producción descontrolada de células inmaduras, que dependiendo del estadio en el que se encuentre la alteración, será la complicación que desarrollará el paciente. A continuación, se observará un cuadro con los diferentes tipos de leucemias agudas de acuerdo a sus variedades morfológicas, según la OMS (Organización Mundial de la Salud) ${ }^{18}$.

Tabla 1. Clasificación de los diferentes tipos de Leucemias Agudas

\begin{tabular}{|c|c|}
\hline Tipo de leucemia & Variedad morfológica \\
\hline $\begin{array}{l}\text { Leucemia aguda mieloide } \\
\text { mínimamente diferenciada } \\
\text { (LMA0) }\end{array}$ & $\begin{array}{l}\text { Como su nombre lo indica, al ser mínimamente diferenciada, quiere decir que las características de sus células tienen } \\
\text { rasgos morfológicos tanto mieloides como linfoides }{ }^{19} \text {. }\end{array}$ \\
\hline Leucemia aguda mieloide (LMA1) & Este tipo de leucemia es sin maduración, lo que indica que hay monomorfismos celulares en sangre periférica ${ }^{18}$. \\
\hline Leucemia aguda mieloide (LMA2) & $\begin{array}{l}\text { Con maduración, en este tipo se observan blastos cuadrangulares; los pacientes presentan una supervivencia más } \\
\text { prolongada y constituyen un subtipo de LMA con anomalías citogenéticas recurrentes, según la clasificación de la } \\
\mathrm{OMS}^{18} \text {. }\end{array}$ \\
\hline Leucemia aguda (LMA3) & $\begin{array}{l}\text { Este LMA Promielocítica es común encontrarla en estado de gestación. Se caracteriza por pro mielocitos malignos que } \\
\text { presentan una translocación de los cromosomas } 15 \mathrm{y} 17^{20} \text {. }\end{array}$ \\
\hline Leucemia aguda (LMA4) & $\begin{array}{l}\text { Mielomonocítica, es un tipo LMA que es más frecuente en niños y jóvenes, se encuentra dentro del grupo de los } \\
\text { síndromes mielodisplásicos y cuenta con un mal pronóstico }{ }^{21} \text {. }\end{array}$ \\
\hline Leucemias agudas (LMA6) & $\begin{array}{l}\text { Las LMA eritroides, significa que hay anomalías en sangre periférica de células de tipo eritroide que están gravemente } \\
\text { afectadas }^{22} \text {. }\end{array}$ \\
\hline Leucemia aguda (LMA7) & $\begin{array}{l}\text { Este tipo de leucemia megacarioblástica, las células se caracterizan por ser de carácter inmaduro, con plaquetas de gran } \\
\text { tamańo }{ }^{23} \text {. }\end{array}$ \\
\hline
\end{tabular}

Fuente: elaboración propia.

58 | Ciencia y Salud 2022; 6(1, enero-abril): 55-64 • Artículo original 


\section{Diagnóstico}

Dentro de los análisis que se utilizan para diagnosticar la LMA, se encuentran los de carácter sanguíneo $\mathrm{y}$ algunos autores coinciden en que realizar una citometría de flujo de una muestra de médula ósea, donde se pueda observar la proliferación mieloide y linfoide que indica la enfermedad. Esta prueba puede llegar a ser muy específica e incluso ayudar a ser diagnósticos diferenciales dentro de las distintas hemopatías que existen ${ }^{24,25}$.

Dentro de la obtención de muestras para el diagnóstico de la LMA están las de médula ósea, que se ejecutan mediante el aspirado de la cresta iliaca o el esternón, a los que posteriormente, para confirmación del diagnóstico, se les realiza con los datos obtenidos de la citomorfología, citoquímica ${ }^{26}$ y análisis inmunofenotípico, donde el $70 \%$ de los casos son diagnosticados, y que se realiza por citometría de flujo, evalúa las proteínas de membrana que expresan los leucocitos dentro de los factores que pronostican. Los datos cromosómicos son una parte importante a evaluar, estas alteraciones citogenéticas pueden presentar un cariotipo complejo en el paciente, que puede llevar al diagnóstico confirmatorio de la leucemia mieloide aguda ${ }^{27}$. Las pruebas hematológicas son fundamentales en este tipo de enfermedades, realizar, por ejemplo, pruebas de químicas (electrolitos, enzimas hepáticas, BUN, calcio y fósforo) y pruebas de coagulación (tiempo parcial de tromboplastina, fibrinógeno, dimero D, tiempo de protombrina) ${ }^{28,29}$.

\section{Aspectos genéticos relacionados con la patología}

Según los estudios que hablan de los análisis citogenéticos en un gran número de pacientes han demostrado que entre el 30 y $50 \%$ de las personas con LMA padecen de traslocaciones e inversiones en los cromosomas somáticos, mientras que solo una minoría tiene una organización de cromosomas somáticos balanceada ${ }^{30}$.
La inv(16)(p13q22) es una alteración que ya está clasificada, según la OMS, como una variación específica para la leucemia mieloide aguda y que además tiene buen pronóstico ${ }^{31}$, junto con otras anomalías que se han venido descubriendo gracias a los estudios en la biología molecular. Dentro de las más recurrentes se encuentran $\mathrm{t}(8 ; 21)$ (q22; q22.1); RUNX1RUNX1T1, inv (16) (p13.1q22) ot (16; 16) (p13.1; q22); CBFB-MYH11, inv (16) (p13.1q22) o t (16; 16) (p13.1; q22); $C B F B-M Y H 11^{32}$, y cada día van siendo más las variaciones a nivel genético que se están analizando, aunque aún no se sabe con certeza si es el componente que más influye en el desarrollo de LAM.

Dentro de las anormalidades de la LMA se encuentra las mutaciones de la proteína NPM1 del exón 12, que en condiciones normales participa en la regulación de las funciones de las moléculas supresoras de tumores, como es el caso de la proteína p53. Se le atribuyen del 35 al $40 \%$ de todos los casos de LMA cuando está mutada. Los pacientes que padecen esta alteración presentan características clínicas y biológicas únicas ${ }^{33,34}$. Sin embargo, esta proteína NPM1 no solo se le atribuye a las alteraciones hematopoyéticas, sino que está relacionado con los tumores sólidos de colon, ovario y vejiga ${ }^{35}$.

Las mutaciones de la NPM1 son marcadores importantes para la LMA por dos factores principales: el primero, porque son los de mayor incidencia en la enfermedad; y segundo, porque ya son considerados marcadores que están en la guía en la clasificación de la Organización Mundial de la Salud (OMS) de 2008, en la sección de la LMA. A partir de esta mutación se pueden analizar varios factores genéticos que permiten descubrir nuevos blancos para el tratamiento de esta alteración hematopoyética tan frecuente en las personas de edad avanzada ${ }^{36,37}$.

\section{Tratamiento}

El trasplante alogénico ha sido un avance en el tratamiento de la LMA, permitiendo cambiar la 
inmunoterapia que se estaba llevando a cabo para este, adicional a las mejoras en la optimización de los procedimientos realizados en el trasplante y, sobre todo, la facilidad de los donantes, que puede ser cualquiera de su familia, siempre y cuando sean compatibles genéticamente; los hermanos constiyuyen un $50 \%$ de los donantes, dada la similitud del HLA (complejo mayor de histocompatibilidad), esto con el fin de evitar el rechazo de quien recibe el tratamiento. Con este procedimiento se permite aumentar resultados positivos de sobrevida libre en los pacientes ${ }^{38,39}$. Esta supervivencia se observa en unos porcentajes hasta casi el $95 \%$, en pacientes que tenían estadios iniciales de la enfermedad y que aun contaban con menos del $5 \%$ de blastos (células hematopoyéticas) en medula ósea ${ }^{40}$.

A pesar de los avances en los últimos años, los resultados de esta alteración han sido bajos; por lo tanto, en estos tiempos se ha podido evidenciar de manera más detallada cómo es el mecanismo de la enfermedad que ha impulsado a nuevas investigaciones para encontrar un buen tratamiento, aún están en fases experimentales los procedimientos como la reparación y transcripción del $\mathrm{ADN}^{41}$.

El tratamiento con quimioterapia convencional es muy efectivo, siempre y cuando se hable de la primera remisión del paciente; sin embargo, los tratamientos, cuando existen recaídas en la leucemia mieloide aguda, son reducidos y con poca efectividad, mostrando un $50 \%$ de respuesta satisfactoria, pues se observa en los pacientes una recuperación parcial o total a nivel hematológico de un 15,3\% con la quimioterapia. Es por esto por lo que algunos estudios confirman que hay una buena respuesta siempre y cuando se realice la quimioterapia de rescate, seguida del trasplante alogénico, ofreciendo mejores resultados de supervivencia global ${ }^{42,43}$.

La terapia de consolidación es frecuentemente usada para mantener el control de la enfermedad, es una quimioterapia que se acompańa del trasplante de células madre hematopoyéticas, conocido como el trasplante alogénico, que puede ser potencialmente curativo en algunos pacientes, se dice "en algunos pacientes" porque se deben tener presentes algunas variables como la edad avanzada, comorbilidades de importancia e incluso el bajo rendimiento ${ }^{44}$, 45 , es por esto que en las últimas décadas se ha visto que este puede ser tratamiento de elección; el trasplante alogénico de células hematopoyéticas hace referencia al paso de células de la médula ósea de un donante compatible genéticamente, que en la mayoría de los casos son los mismos familiares, sin embargo, aún presenta una morbimortalidad elevada (del $25 \%$ aproximadamente), haciendo limitar su uso. El procedimiento que se requiere para el trasplante alogénico es a través de una aspiración de crestas iliacas y, por supuesto, de un Complejo Mayor de Histocompatibilidad (CMH) idéntico ${ }^{46,47}$.

Existen dos tipos principales de trasplante alogénico: uno es el mieloablativo, en el cual se administran dosis altas de quimioterapia, que acaban tanto con las células cancerígenas como con las células de la médula ósea sana; el otro es el no mieloablativo, donde se realiza la quimioterapia con una administración de dosis bajas al paciente, para que este presente una mayor tolerancia y evitando acabar con las células hematopoyéticas sanas ${ }^{48}$. En las personas tratadas con el trasplante alógenico mieloablativo se puede observar una mayor supervivencia de 6,1 meses, respecto a la no mieloablativa que fue de 5,2 ; a pesar de ello, este no fue un resultado muy significativo entre estos dos tipos de tratamiento ${ }^{49}$.

El crecimiento por el uso del tratamiento de trasplante alógenico en la actualidad ha sido muy relevante, porque se puede decir que en el caso del plasma ha venido aumentando aproximadamente de un 1,5 a 9,5 con un promedio del $4 \%$; con lo que cada vez se está reafirmando que el trasplante alogénico podría ser el tratamiento ideal para las hemopatías, específicamente para la $\mathrm{LMA}^{50}$. 


\section{Conclusión}

La LMA es una enfermedad compleja de tratar cuando se encuentra en estadios tardíos y avanzados de la enfermedad, es por eso que en los últimos años ha tomado fuerza la investigación por este tema, donde se ha evidenciado el elevado número de personas alteradas por esta patología. Gracias a estos estudios es que se ha podido encontrar posibles blancos para el tratamiento, cuyos hallazgos ponen en evidencia el aumento de la sobrevida de las personas que la padecen, como ocurre en el caso de los trasplantes alogénicos, que ha tenido una buena respuesta de quienes se someten a este tratamiento. Esto ha abierto paso a nuevos indicios de aumento en la calidad de vida para las personas que padecen LMA, analizando cuál de los trasplantes alogénicos (mieloablativo y no mieloablativo) puede ser la mejor opción.

Esta revisión será de gran utilidad para que haya nuevas investigaciones que puedan descubrir cuál será el mejor tratamiento para este tipo de pacientes y que brinden mucha más información de ayuda para el manejo de esta hemopatía.

Conflicto de intereses: el autor declara no tener conflicto de intereses.

\section{Referencias}

1. Lagunas FA. Leucemia mieloide aguda. Una perspectiva de los mecanismos moleculares del cáncer. Gaceta Mexicana de Oncología, 2016; 15(3):150-7.

2. Ministerio de Salud y Protección Social, Colciencias, Instituto Nacional de Cancerología ESE - Guía de Práctica Clínica para la detección, tratamiento y seguimiento de leucemias linfoblástica y mieloide en población mayor de 18 años. Colombia; 2017.

3. Gómez D, Flores JA, Cantú O, Gutiérrez $\mathrm{CH}$. Utilidad del trasplante de células hematopoyé- ticas en la leucemia mieloide aguda. Rev Hematol Mex, 2012;13(2):74-9.

4. Bhatt VR. Personalizing therapy for older adults with acute myeloid leukemia: Role of geriatric assement and genetic profiling. Anti-Tumour Treatment. 2019;75(1):52-61. Doi: doi.org/10. 1016/j.ctrv.2019.04.001

5. Restrepo AH, Restrepo A, Colorado M, Rivera A, Donado JH. Supervivencia de pacientes con diagnóstico de neoplasias hematológicas admitidos a la Unidad de Cuidado Intensivo del Hospital Pablo Tobón Uribe en Medellín-Colombia entre 2009 y 2014. Acta Colombiana de Cuidado Intensivo, 2017;17(1):14-22. Doi: 10.1016/j.acci.2016.07.005

6. García L, Cabrero M, Del Cañizo C. Leucemias agudas. Medicine. 2016:12(21):1201-12.

7. Blum W, Bloomfield CD. "Leucemia mieloide aguda.”. En Harrison. Principios de Medicina Interna, 20e Jameson J, Fauci AS, Kasper DL, Hauser SL, Longo DL, Loscalzo J. Eds. J. Larry Jameson, et al. New York, NY: McGraw-Hill.

8. Ferlay J, Soerjomataram I, Ervik M, Dikshit R, Eser S, Mathers C, et al. GLOBOCAN 2012 v1.0, Cancer Incidence and Mortality Worldwide

9. Forman D, Bray F, Brewster D, Gombe Mbalawa Ch, Kohler B, Piñeros M, et al. Cancer Incidence in Five Continents, Vol. X (electronic version) Lyon, IARC. http://ci5 iarc fr 2013

10. Combariza JF, Casas CP, Rodríguez M, Cardona AF, Ospina E, Grajales M. Supervivencia en adultos con leucemia linfoide aguda de novo tratados con el esquema HyperCVAD en el Instituto Nacional de Cancerología (Colombia), entre enero de 2001 y junio de 2005. Rev. Colomb Cancerol, 2007;11(2):92-100. 
11. Arrieta E, Jaramillo FJ, Manzia E, Estacio M, Rosales M.C, Donaldo J. Trasplante haploidéntico de progenitores hematopoyéticos en pacientes con diagnóstico de leucemia mieloide aguda. Rev. Colomb. Cancerol. 2019;23(1). Available from: dx.doi.org/10.35509/01239015.74

12. Cranco S, Osorio M, Moirano M.J, Rey N, Funes L, Fischman L, et al. Leucemias Agudas. Sociedad Argentina de Hematología-Guías de diagnóstico y tratamiento; 2017

13. Infante MS, Piris MA, Hernández JA. Alteraciones moleculares en leucemia mieloide aguda y sus implicaciones clínicas y terapéuticas. Med Clin, 2018;151(9):362-7.

14. Moarii M, Papaemmanuil E. Classification and risk assessment in AML: integrating cytogenetics and molecular profiling. Hematology Am Soc Hematol Educ Program, 2017(1):37-44. Available from: doi.org/10.1182/asheducation-2017.1.37

15. Dohner H, Estey EH, Amadori S, et al. European diagnosis and management of acute myeloid leukemia in adults: recommendations from an international expert panel, of behalf of the EuropeanLeukemiaNet. 2010;115(3):453-74. Doi: 10.1182/blood-2009-07-235358

16. Villalba C, Martínez P.A, Acero H. Clinical and epidemiological features of paediatric patients with acute leukaemia in the university clinic, Colombia. Clinical series 2011-2014. Sociedad Colombiana de Pediatría. 2016;49(1):17-22. Available from: doi.org/10.1016/j.rcpe.2016. 01.002

17. Mansouri S, Aractingi I. Manifestaciones cutáneas de las leucemias. EMC-Dermatología. 2004;38(3):1-8. Available from: doi.org/10.1016/ S1761-2896(04)41034-6
18. Gacha MJ, Akle V, Enciso L, Garavito Z. La leucemia linfoblástica aguda y modelos animales alternativos para su estudio en Colombia. Rev. Colomb. Cancerol, 2017;21(4):212-24.

19. Piedras J, Barrales O, López X. Classification of acute leukemias according to the first latin-american consensus conference for the immnophenotyping of leukemias. Rev Invest Clin, 2000; 52(5):524-8.

20. Villamor, N., Zarco, M., Rozman, M. et al. Leucemia mieloblástica aguda con mínima diferenciación mieloide: características fenotípicas y ultraestructurales. Leukemia, 1998;12:1071-5. Doi: 10.1038 / sj.leu.2401074

21. López JM, Fernández E, Contreras M, Bautista A. Manifestaciones de leucemia promielocítica aguda en el embarazo. Progresos de obstetricia y ginecología, 2011;54(8):428-30.

22. Boqueras B, Parada E, Culebras A, Caravaca B, Rives S, Díaz C. Esplenomegalia gigante por leucemia mielomonocítica juvenil. Asociacion Española de Pediatría, ;2009(4):405-7

23. Weinberg O, Seetharam M, Ren L, Seo K, Ma L, Merker JD. et al. Clinical characterization of acute myeloid leukemia with myelodysplasia-related changes as defined by the 2008 WHO classification system. Brief Report, 2009;113(9):1906-8. Available from: doi.org/ 10.1182/blood-2008-10-182782

24. Junca J, Moreno M, Mate JL, Milla F. Diagnóstico simultáneo de una leucemia aguda mieloide y síndrome linforpoliferativo crónico, con amiloidosis AA como episodio final. Medicina Clinica, 2010;135(3):136-7.

25. Morabito L, Raya JM, Hernández L, Hernández MT. Leucemia aguda de células precursoras

62 | Ciencia y Salud 2022; 6(1, enero-abril): 55-64 • Artículo original 
mieloides/ natural killer de presentación simultánea con leucemia linfática crónica. Medicina Clínica. 2008;131(9):356.

26. Yébenes M, Serrano J, Martínez C, Sánchez J. Factores pronósticos clínico-biológicos en pacientes con leucemia aguda mieloblástica en recaída. Medicina Clínica, 2016;147(5):185-91.

27. Bautista L, Martínez H, Bonell O. Hematoma intraparenquimatoso del sistema nervioso central asociado a disatinib en el tratamiento de leucemia mieloide crónica en fase acelerada. Presentación de dos caos y revisión de la literatura. Rev. Colomb. Cancerol.2018. 22(4): $151-156$

28. Blum W, Bloomfield C.D. "Leucemia mieloide aguda." Harrison. Principios de Medicina Interna, 20e Eds. J. Larry Jameson, et al. New York, NY: McGraw-Hill. 2018.

29. Ramos M, López M, Serna J, Ayala R, García L, Barreiro E. Cytogenetic risk categories in acute myeloid leukemia Biomédica Instituto Nacional de Salud 2021 Jun; 41(2): 302-313.

30. Infante MS, Piris MA, Hernández JA. Alteraciones moleculares en leucemia mieloide aguda y sus implicaciones clínicas y terapéuticas. Medicina Clínica, 2018;151(9):362-7. Available from: doi.org/10.1016/j.medcli.2018.05.002

31. Oñate G, Blanco ML, Pratcorona M. Adquisición de la inv(16)(p13q22) en un caso de crisis blástica de leucemia mieloide crónica en tratamiento con imatinib. Medicina Clínica, 2019;152(10):419-20.

32. Arber DA, Orazi A, Hasserjian R, Thiele J, Borowitz MJ, Le Beau MM, et al. The 2016 revision to the World Health Organization classification of myeloid neoplasms and acute leukemia.
Blood, 2016;127(20):2391-405. Available from: doi.org/10.1182/blood-2016-03-643544.

33. Kunchala P, Kuravi S, Jensen R, Mcguirk J, Balusu R. When the good go bad: Mutant NPM1 in acute myeloid leukemia. Blood Reviews. 2018;38(32):167-83.

34. Tang JL, Lin LI, Yao M, Tsay W, Chen CY, et al. Nucleophosmin mutations in de novo acute myeloid leukemia: the age-dependent incidences and the stability during disease evolution. Cancer Res, 2006;66(6):3310-6. Doi: 10. 1158/0008-5472.CAN-05-4316

35. Jeong EG, Lee SH, Yoo NJ, Lee SH. Absence of nucleophosmin 1 (NPM1) gene mutations in common solid cancers. APMIS, 2007;115: 341-6.

36. Alpermann T, Schnittger S, Eder C, Dicker F, Meggendorfer $\mathrm{M}$, et al. Molecular subtypes of NPM1 mutations have different clinical profiles, specific patterns of accompanying molecular mutations and varying outcomes in intermediate risk acute myeloid leukemia. Haematológica, 2016;101(2):55-8. Doi: 10.3324/ haematol. 2015.133819.

37. Verhaak RG, Goudswaard CS, Putten WV, Bijl MA, Sanders MA, Hungens W. Mutations in nucleophosmin (NPM1) in acute myeloid leukemia (AML): association with other gene abnormalities and previously established gene expression signatures and their favorable prognostic significance. Blood, 2005;106(12):3747-54. Available from: doi. org/10.1182/blood-2005-05-2168.

38. Barba P, Elorza I. El trasplante alogénico de progenitores hematopoyéticos en la era de las nuevas terapias en la leucemia linfoblástica aguda. Med Clin, 2019;153(1):28-34. Doi: 10. 1016/j.medcli.2019.01.017 
39. Kebriaei P, Anasetti C, Zhang M.J, Wnag H.L, Aldoss I, Lima M, et al. Intravenous Busulfan Compared with Total Body Irradiation Pretransplant Conditioning for Adults with Acute Lymphoblastic Leukemia, 2018;24(4):726-33. Doi: 10.1016/j.bbmt.2017.11.025

40. Park JH, Riviere I, Gonen M, Wang X, et al. Long-Term Follow-up of CD19 CAR Therapy in Acute Lymphoblastic Leukemia. N Engl J Med, 2018;378(1):449-59. Doi: 10.1056/ NEJMoa1709919

41. LeeDJ,ZeidnerJF.Cyclin-dependentkinase(CDK) 9 ans 4/6 inhibitors in acute myeloid leukemia (AML): a promising therapeutic approach. Expert Opin Investig Drugs, 2019;28(11):989-1001. Doi: 10.1080/13543784.2019.1678583

42. Enciso LJ, Carreño JA, Suárez ML, Bermúdez CD, Arango M, Samudio I, et al. Tratamiento de rescate de leucemia aguda refractaria o en recaída con el régimen IDA-FLAG: experiencia en la rutina de los servicios. Revista Colombiana de Cancerología, 2014;18(2):53-61. Available from: doi.org/10.1016/j.rccan.2014.04.001

43. Perl AE, Martinelli G, Cortes JE, Neubauer A. Gilteritinib or Chemotherapy for Relapsed or Refractory FLT3-Mutated AML. N Engl J Med, 2019;381:1728-40. Doi: 10.1056/ NEJMoa1902688

44. Lyle L, Daver N. Current and emerging therapies for patients with acute myeloid leukemia: a focus on MCL-1 and the CDK9 pathway. Am J Manag Care, 2018;24(16):356-65.

45. Xiang Z, Luo H, Payton JE, et al. La haploinsuficiencia Mcl1 protege a los ratones de la leucemia mieloide aguda inducida por Myc. J Clin Invest, 2010;120 (6):2109-18. Doi: 10. 1172 / JCI39964.
46. Rodríguez LE, Rivera MM. Disminución de mortalidad en hemopatías malignas con un nuevo método de acondicionamiento para trasplante alogénico mieloablativo. Gaceta Mexicana de Oncología. 2016;15(1):16-21. Available from: doi.org/10.1016/j.gamo.2016.02.002

47. Stratmann J, Van E, Rummelt C, Koschade $S$, Rolling C, Lubbert $M$. et al. Low-dose melphalan in elderly patients with relapsed or refractory acute myeloid leukemia: A well-tolerated and effective trearment after hypomethynglating-agent failure, 2019;85. Available from: doi.org/10.1016/j.leukres.2019.106192

48. Sengelov H, Gerds T.A, Braendstrup P, Kornblit B, Mortensen K, Petersen SL, et al. Longterm survival after allogeneic haematopoietic cell transplantation for AML in remission: single-centre results after TBI-based myeloablative and non-myelioablative conditioning. Bone Marrow Transplant. 2013;48(9):1185-91. Doi: $10.1038 / \mathrm{bmt} .2013 .38$

49. Ringden O, Labopin M, Ehninger G, Niederwieser $\mathrm{D}$, Olsson R, Basara N, et al. Reduced intensity conditioning compared with myeloablative conditioning using unrelated donor transplants in patients with acute myeloid leukemia. J Clin Oncol, 2009;27(27):4570-7. Doi: 10.1200/JCO. 2008.20.9692

50. Passweg JR, Baldomero H, Gratwohl A, Bregni M, Cesaro S, Dreger P, de Witte T, Farge-Bancel D, Gaspar B, Marsh J, Mohty M, Peters C, Tichelli A, Velardi A, de Elvira CR, Falkenburg F, Sureda A, Madrigal A; European Group for Blood and Marrow Transplantation (EBMT). The EBMT activity survey: 1990-2010. Bone Marrow Transplant, 2012;47(7):906-23. Doi: 10.1038/bmt.2012.66 